\title{
Lower bounds on the entanglement needed to play XOR non-local games
}

\author{
William Slofstra *
}

July 15, 2010

\begin{abstract}
We give an explicit family of XOR games with $O(n)$-bit questions requiring $2^{n}$ ebits to play near-optimally. More generally we introduce a new technique for proving lower bounds on the amount of entanglement required by an XOR game: we show that near-optimal strategies for an XOR game $G$ correspond to approximate representations of a certain $C^{*}$-algebra associated to $G$. Our results extend an earlier theorem of Tsirelson characterising the set of quantum strategies which implement extremal quantum correlations.
\end{abstract}

\section{Introduction}

The purpose of this paper is to study the amount of entanglement required to play an XOR nonlocal game optimally or near-optimally. In a non-local game, Alice and Bob are asked questions chosen at random according to a known distribution. They win if their answers match the answers required by the game's rules, and lose otherwise. They are physically separated and unable to communicate during the game, so they cannot hope to win every game. Instead, they must meet in advance to determine the best strategy, i.e. one that maximizes the probability that they win. Bell's celebrated theorem [2] states that for some games Alice and Bob can increase their probability of winning if they employ a quantum strategy, in which they make use of an entangled quantum state.

From the viewpoint of quantum computation, a quantum strategy is a means of performing a distributed computation with a higher success probability than what can be achieved classically with no communication [4] 8]. From this viewpoint it is natural to want to know how much entanglement is required to implement an optimal quantum strategy. Entanglement of a pure state can be measured using either the entropy of entanglement (measured in ebits), or the dimension of the underlying Hilbert space at each site; the two measures are related by the fact that any state in $\mathbb{C}^{d} \times \mathbb{C}^{d}$ has at most $\log _{2} d$ ebits. In physics, the importance of lower bounds stems from the fact that non-local games provide empirical evidence that a quantum system is entangled [20]. Lower bounds on the entanglement required by a game imply that the game can be used to verify the degree of entanglement in a quantum system, at least in principle [6].

In this paper we focus on a subset of non-local games generalizing the well-known ClauserHorne-Shomony-Holt (CHSH) game [7]: Alice and Bob each receive exactly one question (drawn from finite sets $S$ and $T$ respectively), each question requires a one-bit answer, and the correct response depends only on the XOR of Alice's and Bob's answers. Non-local games meeting these restrictions are known as XOR non-local games. They have attracted interest in part because of the influential results of Tsirelson [17] [18] which guarantee the existence of an optimal strategy

${ }^{*}$ Department of Mathematics, University of California, Berkeley. Email: slofstra@math.berkeley.edu 
supported on a finite-dimensional Hilbert space, and make it possible to find an optimal strategy using semidefinite programming methods. Tsirelson also proved a lower bound on entanglement for quantum strategies by showing that strategies which implement extreme points of the set of quantum correlations require dimension exponential in the rank of the correlation matrix (see Theorem 2.77).

Tsirelson's lower bound can be dualized (see Proposition 2.6) to show that there are many XOR games requiring dimension exponential in the size of the question sets. In particular nonlocal games exist which are able to verify that quantum states have arbitrarily high dimension. More recent work has readdressed this issue with different methods. Junge, Palazuelos, Perez-Garcia, Villanueva, and Wolf study the ratio $\epsilon(G, m) / \epsilon(G, n)$ for general two and three player non-local games, where $\epsilon(G, d)$ is the maximum success bias for $G$ achievable with a quantum system of dimension $d$ [15] [11]. In particular, they show that two-player games exist for which this ratio is arbitrarily high. However, question sets of size $O\left(2^{d \log ^{2} d}\right)$ are required to verify dimension $d$. Briet, Buhrman, and Toner study a similar ratio for XOR games, where the parameter $d$ in $\epsilon(G, d)$ refers to the rank of the quantum correlation matrix rather than the dimension of the quantum system. The ratio $\epsilon(G, m) / \epsilon(G, n)$ cannot be arbitrarily high for XOR games, since Tsirelson showed that $\epsilon(G, d) / \epsilon(G, 1)$ is always bounded by Grothendieck's constant. Nonetheless, Briet, Buhrman, and Toner show non-constructively that there are XOR games for which this ratio is greater than $1+1 / 2 m+1 / 2 n-O\left(1 / m^{2}\right)$ [5]. Vertesi and Pal give an explicit family of XOR games with question sets of size $O\left(n^{2}\right)$ requiring dimension $n$ to play optimally [19]. Their lower bound is proved by giving a lower bound on the rank of the optimal correlation matrix.

In contrast to the body of work on lower bounds, little is known about upper bounds on entanglement for general non-local games. Tsirelson proved that XOR games with question sets of size $m$ and $n$ require at most $\lfloor r / 2\rfloor$ ebits, where $r$ is the largest integer such that $\left(\begin{array}{l}r \\ 2\end{array}\right)<m+n$. One purpose of this paper is to point out that this lower bound is tight, so XOR games with small question sets can require high entanglement to play optimally. This can be proved by applying Tsirelson's lower bound method to specific families of games, such as the family of examples due to Vertesi and Pal. For completeness, we give another family of examples for which Tsirelson's bound is tight in Section 7. Using a new lower bound technique, we are also able to study the amount of entanglement required by near-optimal strategies. Cleve, Hoyer, Toner, and Watrous showed that an $m \times n$ XOR non-local game has an $\epsilon$-optimal quantum strategy on a Hilbert space of dimension $(m+n+1)^{O\left(\epsilon^{-2}\right)}[8]$. Their proof can be modified to be independent of the size of the question sets, so that fixing $\epsilon$ bounds the entanglement cost for all XOR games (this was apparently first observed by O. Regev, and the author is grateful to R. Cleve and R. Jain for a proof [10]). We show, for a specific family of games, that $\epsilon$-optimal strategies require a Hilbert space of dimension $\min \left(O\left(\epsilon^{-1 / 12}\right), 2^{\lfloor r / 2\rfloor}\right)$, where $r$ is the integer from Tsirelson's upper bound.

The main idea of this paper is to associate to each XOR non-local game a finitely presented $C^{*}$-algebra $\mathcal{A}$ with the property that (a) optimal strategies correspond to representations of $\mathcal{A}$, and (b) near-optimal strategies correspond to approximate representations of $\mathcal{A}$. The presentation for $\mathcal{A}$ depends only on the game rules and a small amount of additional information: the game's marginal biases. Inspiration for this idea comes from the work of Summers and Werner [16] and Tsirelson [17] [18. Summers and Werner show that the Clifford algebra of rank two satisfies property (a) above for the CHSH game. The main statement of Tsirelson's lower bound is that if an XOR non-local game has a unique optimal correlation matrix $C$ then the algebra generated by the observables of any optimal non-degenerate quantum strategy is isomorphic to the Clifford algebra with rank $C$ generators. Our approach is more general, in that it applies to all XOR games, and also to near-optimal strategies. 
The paper is organized as follows. In the next section we give an overview of our results, including the necessary background material and the relation to Tsirelson's results. Marginal biases are introduced in Section 3, and the main result for optimal strategies follows in Section 4 , A link between near-optimal strategies and approximate representations is established in Section 5. In Section 6 we show that the Clifford algebra is stable. Finally, explicit examples are given in Section 7 .

\section{Definitions and statement of results}

\subsection{XOR non-local games and quantum strategies}

The rules for an XOR non-local game are comprised of two finite sets $S$ and $T$ of questions, a probability distribution $\pi$ on $S \times T$, and a function $f: S \times T \rightarrow\{0,1\}$ recording the correct answers. Alice and Bob have access to the rules and may communicate before the game begins. When the game begins, a pair of questions $(i, j)$ is chosen with probability $\pi(i, j)$. Alice receives question $i$, and must output a bit $a$; Bob receives question $j$ and outputs a bit $b$. Alice and Bob win if $a \oplus b$ (the XOR of the two bits) matches $f(i, j)$. All that matters about $S$ and $T$ is their size, so we assume $S=\{1, \ldots, m\}$ and $T=\{1, \ldots, n\}$. The cost matrix for this game is defined to be the $m \times n$ matrix $G$ with $G_{s t}=(-1)^{f(i, j)} \pi(i, j)$. The value of $\pi(i, j)$ can always be recovered from $G_{i j}$, and $f(i, j)$ can also be recovered if $\pi(i, j) \neq 0$. If $\pi(i, j)=0$ then $f(i, j)$ is irrelevant to the game, so an $m \times n$ XOR non-local game is completely described by its cost matrix $G$. A matrix $G$ is the cost matrix of an XOR non-local game if $\sum\left|G_{i j}\right|=1$.

Let $a_{i}$ be the binary random variable corresponding to Alice's output on input $i$, and let $b_{j}$ be the binary random variable corresponding to Bob's output on input $j$. Then $(-1)^{f(i, j)}(-1)^{a_{i}}(-1)^{b_{j}}$ is 1 if Alice and Bob win on question $i, j$, and -1 otherwise. The sum

$$
\sum_{i, j} G_{i j}(-1)^{a_{i}}(-1)^{b_{j}}
$$

is the expected success bias for this behaviour, analagous to the expected success probability, but normalized between -1 and 1.1 In the quantum setting, Alice and Bob are allowed to select a shared bipartite quantum state $|\psi\rangle \in H_{1} \otimes H_{2}$. In addition to the state $|\psi\rangle$, a quantum strategy consists of two families of observables $\left\{A_{i}: i \in S\right\}$ and $\left\{B_{j}: j \in T\right\}$, on the Hilbert spaces $H_{1}$ and $H_{2}$ respectively, with spectra contained in $\{-1,1\}$. The success bias for this quantum strategy is

$$
\sum G_{i j}\left\langle\psi\left|A_{i} \otimes B_{j}\right| \psi\right\rangle
$$

and the quantum success bias $\varepsilon_{q}(G)$ for the game is the maximum success bias across all quantum strategies. The amount of entanglement used by a quantum strategy will be measured using the entropy of entanglement of the state $|\psi\rangle$, which is by definition the von Neumann entropy of the partial trace of $|\psi\rangle$ with respect to $H_{1}$. The dimension of a quantum strategy is $\min \left(\operatorname{dim} H_{1}, \operatorname{dim} H_{2}\right)$.

\section{$2.2 \quad C^{*}$ algebras generated by self-adjoint indeterminates}

Let $R_{1}, \ldots, R_{k}$ be non-commutative polynomials in indeterminates $X_{1}, \ldots, X_{n}$. Let $\mathcal{A}$ be a $C^{*}$ algebra generated by self-adjoint operators $X_{1}, \ldots, X_{n}$. Then $\mathcal{A}$ is said to be the universal $C^{*}$ algebra generated by self-adjoint indeterminates $X_{i}$ satisfying relations $R_{1}, \ldots, R_{k}$ if for all Hilbert spaces $H$ the map $\rho \mapsto\left(\rho\left(X_{1}\right), \ldots, \rho\left(X_{n}\right)\right)$ is a bijection between the representations $\rho$ of $\mathcal{A}$ on

\footnotetext{
${ }^{1}$ If $p$ is the success probability, then the success bias is $2 p-1$.
} 
$H$ and tuples $\left(B_{1}, \ldots, B_{n}\right)$ of bounded self-adjoint operators on $H$ such that $R_{i}\left(B_{1}, \ldots, B_{n}\right)=0$ for all $i=1, \ldots, k$. The universal $C^{*}$-algebra for a set of relations is determined uniquely up to isomorphism, if it exists. The set of relations determining $\mathcal{A}$ is not unique. Since we are interested in $C^{*}$-algebras given by certain relations, we will regard the generators and defining relations as part of the data of the algebra. A universal $C^{*}$-algebra for a given set of relations exists if and only if there are constants $b_{1}, \ldots, b_{n}$ such that $\left\|B_{i}\right\| \leq b_{i}$ whenever $\left(B_{1}, \ldots, B_{n}\right)$ is a tuple of operators satisfying the given relations. For convenience we introduce a slight generalization of the usual notion of a cyclic representation:

Definition 2.1. Let $\mathcal{A}$ be a $C^{*}$-algebra generated by self-adjoint indeterminates $X_{i}$ satisfying relations $R_{j}$. A density-matrix representation of $\mathcal{A}$ is an action of $\mathcal{A}$ on a Hilbert space $H$, determined by a collection of self-adjoint operators $B_{i}$, along with a density matrix $\rho$ on $H$ such that $\mathcal{A} \rho H$ is dense in $H$.

The following universal $C^{*}$-algebra will be used in examples, and in describing previous results of Tsirelson.

Definition 2.2. The Clifford algebra of rank $r$ is the universal $C^{*}$-algebra $\mathcal{C}_{r}$ generated by indeterminates $X_{1}, \ldots, X_{r}$ satisfying the relations $X_{i}^{2}=\mathbb{1}$ for all $1 \leq i \leq n$ and $X_{i} X_{j}=-X_{j} X_{i}$ for all $i \neq j$.

A universal $C^{*}$-algebra generated by $Y_{1}, \ldots, Y_{n}$ is Clifford if for all $1 \leq i, j \leq n$ the anticommutator $Y_{i} Y_{j}+Y_{j} Y_{i}$ is a scalar multiple of the identity. If $\mathcal{A}$ is Clifford with $Y_{i} Y_{j}+Y_{j} Y_{i}=C_{i j} \mathbb{1}$ then $\mathcal{A} \cong \mathcal{C}_{r}$, where $r$ is the rank of the matrix $\left\{C_{i j}\right\}$. The isomorphism can be chosen so that every $Y_{i}$ corresponds to a linear combination of the distinguished generators $X_{j}$ of $C_{r}$. We will call a universal $C^{*}$-algebra strongly Clifford if the Clifford relations $Y_{i} Y_{j}+Y_{j} Y_{i}-V_{i j} \mathbb{1}$ are linear combinations of the defining relations. The representation theory of $C_{r}$ is well-known: $C_{r}$ has either one or two irreducible representations of dimension $2^{\lfloor r / 2\rfloor}$.

\subsection{Main result: algebraic characterization of optimal solutions}

If $\left\{A_{i}\right\},\left\{B_{j}\right\},|\psi\rangle$ is a quantum strategy on a bipartite Hilbert space $H_{1} \otimes H_{2}$, define the marginal (strategy) on $H_{2}$ to be the collection of operators $\left\{B_{j}\right\}$, as well as the density operator $\rho$ which is the partial trace of $|\psi\rangle$ with respect to $H_{1}$. Note that the entanglement of the original strategy is the von Neumann entropy of $\rho$. A strategy $\left\{A_{i}\right\},\left\{B_{j}\right\},|\psi\rangle$ is said to be non-degenerate if there is no projection $P$ commuting with all $A_{i}$ such that that $(P \otimes I)|\psi\rangle=|\psi\rangle$, and no projection $Q$ commuting with all $B_{j}$ such that $(I \otimes Q)|\psi\rangle=|\psi\rangle$. Every degenerate strategy projects down to a unique non-degenerate strategy.

Our main result is a precise description of the marginal strategies corresponding to optimal nondegenerate quantum strategies of a given XOR non-local game $G$. A key part of this description is the fact that for every $m \times n$ XOR non-local game $G$ and $1 \leq i \leq m$ there is a constant $c_{i}$ such that $\sum_{j} G_{i j}\left\langle\psi\left|A_{i} \otimes B_{j}\right| \psi\right\rangle=c_{i}$ whenever $\left\{A_{i}\right\},\left\{B_{j}\right\},|\psi\rangle$ is an optimal quantum strategy. We refer to $c_{i}$ as the $i$ th marginal row bias for $G$. The existence of the marginal biases is proved in Section 3.

If $G$ is an $m \times n$ XOR non-local game with marginal row biases $c_{i}$, define the solution algebra $\mathcal{A}$ for $G$ to be the universal $C^{*}$ algebra generated by self-adjoint indeterminates $X_{1}, \ldots, X_{n}$, satisfying the relations

$$
X_{j}^{2}=\mathbb{1} \text { for all } 1 \leq j \leq n, \text { and }\left(\sum_{j} G_{i j} X_{j}\right)^{2}=c_{i}^{2} \cdot \mathbb{1} \text { for all } 1 \leq i \leq m .
$$


Theorem 2.3. Let $G$ be a XOR non-local game with no zero rows or columns and solution algebra A. A collection of bounded linear operators $\left\{B_{j}: j \in T\right\}$ and density operator $\rho$ on a Hilbert space $H$ is the marginal of a non-degenerate optimal strategy for $G$ if and only if the map $X_{j} \mapsto B_{j}$ induces a density-matrix representation of $\mathcal{A}$ on $H$ with density matrix $\rho$, and $\rho$ commutes with the image of $\mathcal{A}$.

Theorem 2.3 determines the entanglement required by $G$ in the following sense:

Corollary 2.4. Let $G$ be an XOR non-local game with no zero rows or columns, and let $\mathcal{A}$ be the corresponding solution algebra. Let $N$ be the minimum dimension among non-zero representations of $\mathcal{A}$. Then the minimum entanglement used by an optimal quantum strategy for $G$ is $\log _{2} N$. The strategies which attain the minimum entanglement are the irreducible representations of $\mathcal{A}$ of dimension $N$, with cyclic state $I / N$, where I denotes the identity operator.

The dimension of a representation is the dimension of the underlying Hilbert space. Although the results above are stated for pure states, Corollary 2.4 extends trivially to mixed states when the entanglement of formation is used as an entanglement measure. A mixed state of minimum entanglement must be a mixture of maximally entangled states. The proofs of Theorem 2.3 and Corollary 2.4 are given in Section 4 .

\subsection{Comparison to Tsirelson's results}

An $m \times n$ matrix $\left\{c_{i j}\right\}$ is called a quantum correlation if there is a quantum strategy $\left\{A_{i}\right\},\left\{B_{j}\right\},|\psi\rangle$ such that $c_{i j}=\left\langle\psi\left|A_{i} \otimes B_{j}\right| \psi\right\rangle$ for all $i, j$. The set $C_{m n}$ of all $m \times n$ quantum correlations is closed and convex, so the problem of finding the quantum success bias for a game $G$ can be formulated as the following convex programming problem:

$$
\max \sum_{i, j} G_{i j} c_{i j}: \text { where }\left\{c_{i j}\right\} \text { is a quantum correlation. }
$$

The optimisation problem $(\Phi)$ can be formulated as a semidefinite programming problem using the following theorem. This makes it possible to find a near optimal quantum correlation in practice.

Theorem 2.5 (Tsirelson, 1987). An $m \times n$ matrix $\left\{c_{i j}\right\}$ is a quantum correlation matrix if and only if there are two families of vectors $\left\{u_{i} \in \mathrm{B}\left(\mathbb{R}^{N}\right): i \in S\right\}$ and $\left\{v_{j} \in \mathrm{B}\left(\mathbb{R}^{N}\right): j \in T\right\}$ such that $c_{i j}=u_{i} \cdot v_{j}$.

Here $\mathrm{B}\left(\mathbb{R}^{N}\right)$ denotes the unit ball in $\mathbb{R}^{N}$. Define a vector strategy for $\left\{c_{i j}\right\}$ to be two collections of vectors $\left\{u_{i}\right\}$ and $\left\{v_{j}\right\}$ in $\mathrm{B}\left(\mathbb{R}^{N}\right)$ such that $c_{i j}=u_{i} \cdot v_{j}$. The proof of Theorem 2.5 is constructive, associating to a vector strategy a quantum strategy $\left\{A_{i}\right\},\left\{B_{j}\right\},|\psi\rangle$ with the same correlation. The observables $\left\{A_{i}\right\}$ and $\left\{B_{j}\right\}$ are constructed using the Clifford algebra $C_{N}$, and consequently Theorem 2.5 implies that every $m \times n$ game has an optimal quantum strategy using $\lfloor n / 2\rfloor$ ebits. Tsirelson [17] showed that this upper bound could be improved to $\lfloor r / 2\rfloor$ ebits, where $r$ is the largest integer such that $\left(\begin{array}{c}r+1 \\ 2\end{array}\right)<m+n$.

Proposition 2.6. For every $n \geq 2$ there is an $m \times n$ XOR game for which Tsirelson's bound is tight.

Proof. Theorem 2.22 of [18] can be used to find a maximal rank extreme point of the set of quantum correlations. Any supporting hyperplane for this extreme point will satisfy the proposition.

For a constructive proof, apply either Theorem 2.3 or Theorem 2.7 below to explicit examples, such as the family of games in Section $7\left(m=\left(\begin{array}{l}n \\ 2\end{array}\right)\right)$ or the similar family of games in [19] $(m=$ $\left.\left(\begin{array}{l}n \\ 2\end{array}\right)+1\right)$. 
There is a strong relation between Theorem 2.3 and the following theorem of Tsirelson.

Theorem 2.7 (Tsirelson [17]). Suppose that $\left\{c_{i j}\right\}$ is an extreme point of $C_{m n}$ of rank $r$, and $\left\{A_{i}\right\}$, $\left\{B_{j}\right\},|\psi\rangle$ is a non-degenerate strategy ${ }^{2}$ representing $\left\{c_{i j}\right\}$. Then the strategy $\left\{A_{i}\right\},\left\{B_{j}\right\},|\psi\rangle$ is Clifford, and $|\psi\rangle$ uses at least $\lfloor r / 2\rfloor$ ebits.

One way of understanding Tsirelson's theorem is that it associates a Clifford algebra to every extreme point of $C_{m n}$. XOR non-local games are in one-to-one correspondence with supporting hyperplanes of $C_{m n}$ via the cost matrix, so Theorem 2.3 describes how to associate a $C^{*}$-algebra to every supporting hyperplane of $C_{m n}$. Thus the two results are complementary, and correspond to two approaches to describing convex sets: by extreme points and by supporting hyperplanes. Furthermore, we now have two ways of showing that an XOR non-local game requires high entanglement: if the representations of the solution algebra are known then we can apply Corollary 2.4. and if we can show that the optimization problem $(\Phi)$ has a unique solution of high rank then this solution must be an extreme point of $C_{m n}$ and we can apply Theorem 2.7. Both approaches work for the examples of games requiring high entanglement presented in Section 7.

This raises the question of whether Theorems 2.3 and 2.4 tell us anything new about optimal strategies and entanglement requirements, especially since $\mathcal{A}$ is almost always Clifford. The answer is yes, for interesting reasons. Theorem 2.7 does not give a full description of the optimal strategies for a game $G$ unless $(\Phi)$ has a unique solution.

Proposition 2.8. The solution algebra $\mathcal{A}$ for a game $G$ with no zero rows or columns is Clifford if and only if there is a unique quantum correlation which is optimal for $G$.

Proof. For $\mathcal{A}$ to be Clifford means that $X_{i} X_{j}+X_{j} X_{i}=2 V_{i j} \mathbb{1}$ for all $i, j$ and some fixed $n \times n$ matrix $V$. In Proposition 3.3 , we will give an optimization problem $(\Gamma)$ with the property that $X_{i} X_{j}+X_{j} X_{i}=2 V_{i j} \mathbb{1}$ in some representation of $\mathcal{A}$ if and only if $V$ is an optimal solution to $(\Gamma)$. When $G$ has no zero rows or columns there is a one-to-one relationship between optimal solutions of $(\Gamma)$ and optimal quantum correlations. If $\mathcal{A}$ is Clifford then $(\Gamma)$ will have a unique solution, so there must be a unique optimal quantum correlation.

On the other hand, if there is a unique quantum correlation, then $(\Gamma)$ will also have a unique solution. By Theorem 2.7, the relation $X_{i} X_{j}+X_{j} X_{i}=2 V_{i j} \mathbb{1}$ will hold in every representation of $\mathcal{A}$, with $V$ the unique solution to $(\Gamma)$, so this relation holds in $\mathcal{A}$ itself.

In addition, the strategy of minimum entanglement is not always Clifford.

Proposition 2.9. For any $n \geq 1$ there is a XOR non-local game such that any optimal strategy which is Clifford uses at least $n$ ebits more than the strategy of minimum entanglement. For an example, see the game $C L(n)$ described in Section 7 .

In particular, the correlation of a strategy of minimum entanglement does not have to be an extreme point of $C_{m n}$, and the minimum entanglement required by a game is not a function of the rank of the optimal quantum correlation.

\subsection{Stability and approximate strategies}

Stability questions arise naturally in applications of Theorem 2.3 , For example, suppose we only have an approximation $\hat{c}_{i}$ to the marginal biases $c_{i}$. Are the representations of the algebra deter-

\footnotetext{
${ }^{2}$ Tsirelson actually proves this result for a slightly different definition of quantum strategy, but the result still holds for the definition in use here.
} 
mined by the relations

$$
X_{j}^{2}=\mathbb{1} \text { for all } 1 \leq j \leq n, \text { and }\left(\sum_{j} G_{i j} X_{j}\right)^{2}=\hat{c}_{i}^{2} \cdot \mathbb{1} \text { for all } 1 \leq i \leq m .
$$

close to representations of the solution algebra $\mathcal{A}$ ? This question is related to the study of stable relations for $C^{*}$-algebras, and the broader area of Hyers-Rasiass-Ulam stability in functional analysis and group theory. We use the following definition of stability due to [13] - note that a norm condition $\left\|B_{i}\right\| \leq\left\|X_{i}\right\|_{\mathcal{A}}$ is added to simplify some of the later analysis.

Definition 2.10. Let $\mathcal{A}$ be a universal $C^{*}$-algebra generated by self-adjoint indeterminates $X_{1}, \ldots, X_{n}$ satisfying relations $R_{1}, \ldots, R_{k}$. An $\epsilon$-approximate representation of $\mathcal{A}$ is a collection of bounded self-adjoint operators $B_{1}, \ldots, B_{n}$ on a Hilbert space $H$, such that $\left\|B_{i}\right\| \leq\left\|X_{i}\right\|_{\mathcal{A}}$ for all $1 \leq i \leq n$ and $\left\|R_{i}\left(B_{1}, \ldots, B_{n}\right)\right\| \leq \epsilon$ for all $1 \leq i \leq k$.

$A$ set of relations $R_{1}, \ldots, R_{k}$ is stable (for finite-dimensional Hilbert spaces) if there is a constant $\delta>0$ and function $f:[0, \delta) \rightarrow[0,+\infty)$ such that if $0<\epsilon<\delta$ and $\left(B_{1}, \ldots, B_{n}\right)$ is an $\epsilon$-approximate represention of $\mathcal{A}$ on a finite-dimensional Hilbert space $H$ then there is a representation $B_{1}^{\prime}, \ldots, B_{n}^{\prime}$ of $\mathcal{A}$ on $H$ with $\left\|B_{i}-B_{i}^{\prime}\right\| \leq f(\epsilon)$.

A note on norms: we use $\|\cdot\|$ to denote the operator norm induced by the Hilbert space structure. On finite-dimensional Hilbert spaces we can also use the Frobenius norm $\|X\|_{F}=\sqrt{\operatorname{tr}\left(X^{*} X\right)}$. The two norms are related by the inequality $\|\cdot\| \leq\|\cdot\|_{F} \leq \sqrt{d}\|\cdot\|$, where $d$ is the dimension.

\section{Proposition 2.11.}

- The Clifford algebra $C_{r}$ is stable with constant $\delta=1 /\left(250 r^{2}\right)$ and function $f(\epsilon)=5 r^{2} \epsilon / 2$.

- If $\mathcal{A}$ is strongly Clifford of rank $r$ then there is a constant $\delta>0$, such that if $\mathcal{A}$ has an $\epsilon$ approximate representation with $\epsilon<\delta$ then there is an exact representation of $\mathcal{A}$ of the same dimension.

- Almost all $m \times n$ games with $m \geq\left(\begin{array}{l}n \\ 2\end{array}\right)$ are strongly Clifford.

The proof of Proposition 2.11 is given in Section 6. Although we do not make use of the fact, the solution algebra of an $m \times n$ game is stable if it is strongly Clifford of rank $n$. The author does not know whether or not the relations for the solution algebra are stable in general.

A quantum strategy is said to be $\epsilon$-optimal for a game $G$ if it has success bias within $\epsilon$ of $\varepsilon_{q}(G)$. It is possible to use the solution algebra to study $\epsilon$-optimal strategies as $\epsilon \rightarrow 0$. It will be clear from the proof of Theorem 2.3 that an approximate representation of $\mathcal{A}$ will be the marginal of a near-optimal strategy for $G$. A weak converse is also true.

Theorem 2.12. For every $m \times n$ XOR-non-local game with no zero rows or columns there are constants $C, C^{\prime}>0$ such that if $0<\epsilon<C$ and $\left\{B_{j}: j \in T\right\}, \rho$ is the marginal of an $\epsilon$-optimal strategy supported on a Hilbert space $H$ with $\operatorname{dim} H=d<+\infty$, then there is a projection $P$ on $H$ such that $\left\{P B_{j} P\right\}$ is a $\left(C^{\prime} d^{3 / 2} \epsilon^{1 / 8}\right)$-approximate representation for the solution algebra of $G$.

Specifically, $C \geq \min _{j} d_{j}^{16} /(100(m+n))\left(d_{j}\right.$ is the $j$ th marginal column bias $)$, and $C^{\prime} \leq 15(m+$ $n)^{\frac{1}{8}}$. The proof of this theorem is given in Section 5 .

For near-optimal strategies we would like to understand the dimensions of the Hilbert spaces supporting $\epsilon$-optimal strategies, for $\epsilon$ in a neighbourhood of zero. For this to make sense, we look at 
finite-dimensional spaces only. Cleve, Hoyer, Toner, and Watrous have shown that an $m \times n$ XOR non-local game has an $\epsilon$-optimal quantum strategy on a Hilbert space of dimension $(m+n+1)^{O\left(\epsilon^{-2}\right)}$ [8]. Regev has pointed out that this bound can be made independent of $m$ and $n$, so that fixing the precision $\epsilon$ upper bounds the entanglement cost for all XOR non-local games [10]. Theorem 2.12 gives lower bounds on the dimension of approximate solutions when the solution algebra $\mathcal{A}$ is strongly Clifford.

Corollary 2.13. Let $G$ be an XOR non-local game with no zero rows or columns, and solution algebra $\mathcal{A}$ which is strongly Clifford of rank $r$. Then there are constants $C$ and $C^{\prime \prime}$ such that if $0<\epsilon \leq C$ then any $\epsilon$-optimal strategy is supported on a Hilbert space of dimension at least $\min \left(C^{\prime \prime} \epsilon^{-1 / 12}, 2^{\lfloor r / 2\rfloor}\right)$.

Proof. Let $C, C^{\prime}$ to be the constants from Theorem 2.12. Suppose $G$ has an $\epsilon$-optimal solution on a Hilbert space $H$ of dimension $d, 0 \leq \epsilon \leq C$. By Theorem [2.12, $\mathcal{A}$ has a $C^{\prime} d^{3 / 2} \epsilon^{1 / 8}$-approximate representation of dimension $d$. Take $C^{\prime \prime}=\left(C^{\prime} / \delta\right)^{-2 / 3}$, where $\delta$ is the constant from Proposition 2.11. If $d<C^{\prime \prime} \epsilon^{-1 / 12}$ then

$$
C^{\prime} d^{3 / 2} \epsilon^{1 / 8}<\delta
$$

so Proposition 2.11 implies that $\mathcal{C}_{r}$ has an exact representation on $H$, implying that $d \geq 2^{\lfloor r / 2\rfloor}$.

The lower bound in Corollary 2.13 appears to be quite weak. For instance, it is possible to show that dimension increases linearly with $1-\epsilon$ up to dimension $n$, for a specific family of games with question sets of size $n(n-1)$ and $n$ (see Proposition 7.2).

\section{Marginal biases}

Theorem 3.1. For every $m \times n X O R$ non-local game $G$ there is a collection of non-negative constants $\left\{c_{i}: i \in S\right\}$ such that if a vector strategy $\left\{u_{i}\right\},\left\{v_{j}\right\}$ for $G$ is $\epsilon$-optimal, $0 \leq \epsilon<$ $1 /(4(m+n))$, then

$$
\left\|\sum_{j} G_{i j} v_{j}-c_{i} u_{i}\right\| \leq \sqrt{10}(m+n)^{1 / 4} \epsilon^{1 / 4} .
$$

If $G$ has no zero rows then the constants $c_{i}$ are non-zero.

When $\epsilon=0, \sum_{j} G_{i j} v_{j}=c_{i} u_{i}$, so the sums $\sum_{g} G_{i j} u_{i} \cdot v_{j}$ are constant (and equal to $c_{i}$ ) for every optimal vector strategy $\left\{u_{i}\right\},\left\{v_{j}\right\}$. There are also marginal column biases $d_{j}$ playing the same role for the columns. The marginal column biases are non-zero when $G$ has no zero columns.

Corollary 3.2. Let $G$ be an XOR non-local game with no zero rows. A vector strategy $\left\{u_{i}\right\},\left\{v_{j}\right\}$ for $G$ is optimal if and only if

$$
\sum_{j} G_{i j} v_{j}=c_{i} u_{i} \text { for all } 1 \leq i \leq m,
$$

where $c_{i}$ is the ith marginal row bias of $G$.

Alternatively, we can characterize optimal vector strategies without any reference to the vectors $u_{i}$ at all. 
Corollary 3.3. Let $\left\{v_{j}\right\}$ be a collection of $n$ unit vectors. Then there are unit vectors $\left\{u_{i}\right\}$ such that the quantum correlation $\left\{u_{i} \cdot v_{j}\right\}$ is an optimal solution to $G$ if and only if the positive semidefinite matrix $\left\{v_{i} \cdot v_{j}\right\}$ is an optimal solution to

$$
\max \sum_{i} \sqrt{\sum_{j, k} G_{i j} G_{i k} V_{j k}}: V \text { is an } n \times n \text { positive semidefinite matrix with } V_{i i}=1 \text {. }
$$

If $G$ has no zero rows or columns there is a one-to-one relationship between solutions to ( $\Gamma)$ and optimal quantum correlations for $G$. If $V$ is an optimal solution then the ith marginal row bias is given by $c_{i}^{2}=\sum_{j, k} G_{i j} G_{i k} V_{j k}$.

To prove Theorem 3.1, consider the semidefinite programming (SDP) formulation for optimal value of $G$ given in 9 . Specifically, let

$$
B=\left(\begin{array}{cc}
0 & \frac{1}{2} G \\
\frac{1}{2} G^{T} & 0
\end{array}\right)
$$

Then $\varepsilon_{q}(G)$ is equal to

$$
\text { (P) } \quad \max \operatorname{tr}(B X): X_{i i}=1 \text { for } 1 \leq i \leq m+n \text {, and } X \succeq 0,
$$

where $\succeq$ denotes the partial order induced by the semidefinite cone. An overview of semidefinite programming techniques can be found in [3]. There is a direct correspondence between vector strategies and feasible solutions of $(P)$ : if $\left\{u_{i}\right\},\left\{v_{j}\right\}$ is a vector strategy, $U$ is the matrix with columns given by the vectors $u_{i}$, and $V$ is the matrix with columns given by the vectors $v_{j}$, then the corresponding feasible solution to $(P)$ is the matrix

$$
X=\left(\begin{array}{l}
U^{T} \\
V^{T}
\end{array}\right) \cdot\left(\begin{array}{ll}
U & V
\end{array}\right)=\left(\begin{array}{ll}
U^{T} U & U^{T} V \\
V^{T} U & V^{T} V
\end{array}\right)
$$

and this construction can be run in reverse as well. The success bias can also be calculated using the dual formulation:

$$
\begin{array}{r}
\min \frac{1}{2} \sum_{1 \leq i \leq m} c_{i}+\frac{1}{2} \sum_{1 \leq i \leq n} d_{j}: \\
\frac{1}{2}\left(\begin{array}{cc}
\Delta(c) & 0 \\
0 & \Delta(d)
\end{array}\right) \succeq B .
\end{array}
$$

Here $\Delta(c)$ denotes the diagonal matrix with the entries of $c$ on the diagonal.

Proof of Theorem 3.1. Let $c_{1}, \ldots, c_{m}, d_{1}, \ldots, d_{n}$ be optimal for $(D)$, and let

$$
S=\frac{1}{2}\left(\begin{array}{cc}
\Delta(c) & 0 \\
0 & \Delta(d)
\end{array}\right)-B
$$

Let $X$ be the positive semidefinite matrix corresponding to a (not necessarily optimal) vector strategy $\left\{u_{i}\right\},\left\{v_{j}\right\}$. Then

$$
(S X)_{i i}=\left\{\begin{array}{ll}
\frac{1}{2} c_{i}-\frac{1}{2} \sum_{j} G_{i j} u_{i} \cdot v_{j} & 1 \leq i \leq m \\
\frac{1}{2} d_{i-m}-\frac{1}{2} \sum_{j} G_{j(i-m)} u_{j} \cdot v_{i-m} & m+1 \leq i \leq m+n
\end{array},\right.
$$


and in particular

$$
\operatorname{tr}(S X)=\frac{1}{2} \sum_{1 \leq i \leq m} c_{i}+\frac{1}{2} \sum_{1 \leq j \leq n} d_{j}-\operatorname{tr}(B X)=\varepsilon_{q}(G)-\operatorname{tr}(B X),
$$

where the last equality holds by strong duality. The vector strategy in question is $\epsilon$-optimal if and only if $X$ is $\epsilon$-optimal for $(P)$, meaning that $\varepsilon_{q}(G)-\operatorname{tr}(B X) \leq \epsilon$. The following inequality holds for every $1 \leq i \leq m$ and every $\epsilon$-optimal vector strategy:

$$
\begin{aligned}
\left|c_{i}-\sum_{j} G_{i j} u_{i} \cdot v_{j}\right| & =2\left|(S X)_{i i}\right| \leq 2\|S X\|_{F} \leq 2\left\|X^{1 / 2}\right\|_{F}\left\|S^{1 / 2}\right\|_{F}\left\|X^{1 / 2} S^{1 / 2}\right\|_{F} \\
& =2 \sqrt{\operatorname{tr}(X)} \sqrt{\operatorname{tr}(S)} \sqrt{\operatorname{tr}(X S)} \leq 2 \sqrt{m+n} \sqrt{\varepsilon_{q}(G)} \sqrt{\epsilon}
\end{aligned}
$$

where $\|\cdot\|_{F}$ is the Frobenius norm. Let $\delta=2 \sqrt{m+n} \sqrt{\varepsilon_{q}(G)} \sqrt{\epsilon}$. Then

$$
\left\|c_{i} u_{i}-\sum_{j} G_{i j} v_{j}\right\|^{2}=c_{i}^{2}-2 c_{i} \sum_{j} G_{i j} u_{i} \cdot v_{j}+\left\|\sum_{j} G_{i j} v_{j}\right\|^{2} .
$$

We know that $\sum_{j} G_{i j} u_{i} \cdot v_{j}>c_{i}-\delta$. To bound $\left\|\sum_{j} G_{i j} v_{j}\right\|$, let $u_{i}^{\prime}$ be the vector $\sum_{j} G_{i j} v_{j}$, renormalized to a unit vector. Then

$$
\sum_{j} G_{i j} u_{i}^{\prime} \cdot v_{j}=\left\|\sum_{j} G_{i j} v_{j}\right\| \geq \sum_{j} G_{i j} u_{i} \cdot v_{j}
$$

so $\left\{u_{i}^{\prime}\right\},\left\{v_{j}\right\}$ is also an $\epsilon$-optimal strategy for $G$. Hence $\left\|\sum_{j} G_{i j} v_{j}\right\|=\sum_{j} G_{i j} u_{i}^{\prime} \cdot v_{j} \leq c_{i}+\delta$. We can conclude that

$$
\left\|c_{i} u_{i}-\sum_{j} G_{i j} v_{j}\right\|^{2} \leq c_{i}^{2}-2 c_{i}\left(c_{i}-\delta\right)+\left(c_{i}+\delta\right)^{2}=4 c_{i} \delta+\delta^{2},
$$

and this last expression is at most $5 \varepsilon_{q}(G)^{1 / 2} \delta$ if $\epsilon \leq 1 /(4(m+n))$. Since $\epsilon_{q}(G) \leq 1$, this shows that the constants $c_{i}$ satisfy the statement of Theorem 3.1. Uniqueness follows from the fact that $c_{i}=\sum_{j} G_{i j} u_{i} \cdot v_{j}$ for every optimal vector strategy $\left\{u_{i}\right\},\left\{v_{j}\right\}$. Finally $S$ is positive semidefinite, so if the $i$ th row of $G$ is non-zero then $c_{i}$ must be strictly positive.

\section{Quantum strategies: the exact case}

We begin with the proof of Theorem 2.3. For this we introduce some notation, which we will keep fixed for this and also the next section. Suppose $|\psi\rangle$ is an element of $H_{1} \otimes H_{2}$. If we pick a basis $|i\rangle$ for $H_{1}$ then we can write $|\psi\rangle=\sum|i\rangle \lambda|i\rangle$, where $\lambda: H_{1} \rightarrow H_{2}$ is a linear transformation. With this notation, the Schmidt coefficients for $|\psi\rangle$ are the eigenvalues of $\lambda$, and the partial trace of $|\psi\rangle$ with respect to $H_{1}$ is $\rho=\lambda \lambda^{*}$, a density operator on $H_{2}$. Having picked a basis on $H_{1}$, we can write any element of $\mathcal{B}\left(H_{1}\right)$ as a matrix. If $A \in \mathcal{B}\left(H_{1}\right)$, let $\bar{A}$ denote the linear transformation constructed by taking the entry-wise complex conjugate of the matrix of $A$. Note that the operation $A \mapsto \bar{A}$ depends on the choice of basis for $H_{1}$. 
Lemma 4.1. Let $A$ and $B$ be Hermitian operators on Hilbert spaces $H_{1}$ and $H_{2}$ respectively. Then $\|(A \otimes \mathbb{1}-\mathbb{1} \otimes B)|\psi\rangle\|=\| \lambda \bar{A}-B \lambda \|_{F}$. Consequently, if $\|(A \otimes \mathbb{1}-\mathbb{1} \otimes B)|\psi\rangle \| \leq \epsilon$, then $\|\rho B-B \rho\|_{F} \leq 2 \epsilon$, or in other words $\rho$ approximately commutes with $B$.

Proof. For the first identity, observe that $(A \otimes \mathbb{1})|\psi\rangle=\sum_{i}|i\rangle \lambda \bar{A}|i\rangle$, while $(\mathbb{1} \otimes B)|\psi\rangle=\sum_{i}|i\rangle B \lambda|i\rangle$. So $\|(A \otimes \mathbb{1}-\mathbb{1} \otimes B)|\psi\rangle\left\|^{2}=\right\| \lambda \bar{A}-B \lambda \|_{F}^{2}$.

If $\|(A \otimes \mathbb{1}-\mathbb{1} \otimes B)|\psi\rangle \| \leq \epsilon$ then $\left\|B \rho-\lambda \bar{A} \lambda^{*}\right\|_{F} \leq\|B \lambda-\lambda \bar{A}\|_{F}\left\|\lambda^{*}\right\|_{F} \leq \epsilon$. But $\lambda \bar{A} \lambda^{*}$ is Hermitian, while $(B \rho)^{*}=\rho B$.

Now we should say something about what it means for an operator strategy to be nondegenerate. Suppose that $\left\{A_{i}\right\},\left\{B_{j}\right\},|\psi\rangle$ is a quantum strategy, and let $\mathcal{B}_{1}$ and $\mathcal{B}_{2}$ denote the $C^{*}$-algebras generated by $A_{1}, \ldots, A_{m}$ and $B_{1}, \ldots, B_{n}$ respectively. Let cl denote the closure of a set in a Hilbert space with respect to the norm topology.

Lemma 4.2. The strategy in question is non-degenerate if and only if $\operatorname{cl} \mathcal{B}_{2} \lambda H_{1}=H_{2}$ and $\operatorname{cl} \overline{\mathcal{B}}_{1} \lambda^{*} H_{2}=$ $H_{1}$.

Proof. Let $Q$ be a projection onto a closed subspace $W$ of $H_{2}$. By Lemma 4.1. $(I \otimes Q)|\psi\rangle=|\psi\rangle$ if and only if $Q \lambda=\lambda$, and this occurs if and only if the image of $\lambda$ is contained in $W$. Also $Q$ will commute with $\mathcal{B}_{2}$ if and only if $W$ is an invariant subspace for $\mathcal{B}_{2}$. Thus there is a projection $Q$ commuting with $\mathcal{B}_{2}$ such that $(I \otimes Q)|\psi\rangle=|\psi\rangle$ if and only if $\operatorname{cl} \mathcal{B}_{2} \lambda H_{1}$ is a strict subset of $H_{2}$.

There is a projection $P$ such that $(P \otimes I)|\psi\rangle=|\psi\rangle$ if and only if $\lambda \bar{P}=\lambda$, and this is equivalent to the condition that $\bar{P} \lambda^{*}=\lambda^{*}$ (note that $\bar{P}$ is also a projection). $P$ will commute with $\mathcal{B}_{1}$ if and only $\bar{P}$ commutes with $\overline{\mathcal{B}}_{1}$. Thus we arrive at the requirement that $\operatorname{cl} \overline{\mathcal{B}}_{1} \lambda^{*} H_{2}=H_{1}$.

From this lemma it is easy to see that every strategy projects down to a unique non-degenerate strategy. One more special case is important. The kernel of $\lambda$ is the orthogonal complement of the image of $\lambda^{*}$, so $\operatorname{cl} \lambda H_{1}=\operatorname{cl} \rho H_{2}$. If $\rho$ commutes with $\mathcal{B}_{2}$ then $\rho H_{2}$ is an invariant subspace of $\mathcal{B}_{2}$, so $\mathrm{cl} \mathcal{B}_{2} \lambda H_{1}=H_{2}$ if and only if $\operatorname{cl} \rho H_{2}=H_{2}$.

Proof of Theorem [2.3. Suppose we are given an optimal non-degenerate strategy $\left\{A_{i}\right\},\left\{B_{j}\right\},|\psi\rangle$ for $G$, acting on Hilbert spaces $H_{1}$ and $H_{2}$. We fix a basis for $H_{1}$, and use the notation introduced above for $|\psi\rangle$. Define $u_{i}=\left(A_{i} \otimes I\right)|\psi\rangle$ and $v_{j}=\left(I \otimes B_{j}\right)|\psi\rangle$. The vectors $u_{1}, \ldots, u_{m}$ and $v_{1}, \ldots, v_{n}$ form an optimal vector strategy for $G$. Let $c_{i}$ denote the $i$ th marginal row bias for $G$, and $d_{j}$ the $j$ th marginal column bias. By Corollary [3.2, we know that

$$
d_{j}\left(I \otimes B_{j}\right)|\psi\rangle=\sum_{i} G_{i j}\left(A_{i} \otimes I\right)|\psi\rangle
$$

where each $d_{j}$ is non-zero. It follows from Lemma 4.1 that $\rho$ commutes with all $B_{j}$. As previously mentioned, Lemma 4.2 now implies that the closure of the image of $\rho$ is equal to $\mathrm{H}_{2}$.

Now we also know that

$$
c_{i}\left(A_{i} \otimes I\right)|\psi\rangle=\sum_{j} G_{i j}\left(I \otimes B_{j}\right)|\psi\rangle
$$

and consequently by Lemma $4.1 \sum_{j} G_{i j} B_{j} \lambda=c_{i} \lambda \bar{A}_{i}$. We apply the map $S \mapsto S S^{*}$ to both sides to get

$$
\left(\sum_{j} G_{i j} B_{j}\right)^{2} \rho=\left(\sum_{j} G_{i j} B_{j}\right) \rho\left(\sum_{j} G_{i j} B_{j}\right)=c_{i}^{2} \lambda \bar{A}_{i}{ }^{2} \lambda^{*}=c_{i}^{2} \rho .
$$


Since the closure of the image of $\rho$ is equal to $H_{2}$, we find that $\left(\sum_{j} G_{i j} B_{j}\right)^{2}=c_{i}^{2} I$. Thus the operators $\left\{B_{j}\right\}, \rho$ describe a cyclic representation of $\mathcal{A}$, and the density operator $\rho$ commutes with $\mathcal{A}$.

Conversely, suppose we are given a cyclic representation $\left\{B_{j}\right\}, \rho$ of $\mathcal{A}$, acting on a Hilbert space $H$, such that $\rho$ commutes with all $B_{i}$ and $\operatorname{cl} \mathcal{A} \rho H=H$. We can conclude that $\operatorname{cl} \rho H=H$, so if $\lambda$ is the square root of $\rho$, then $\rho H \subset \lambda H$, and hence $\operatorname{cl} \lambda H=H$. Now set $H_{1}=H_{2}=H$ and $|\psi\rangle=\sum_{i}|i\rangle(\lambda|i\rangle)$. Define $\bar{A}_{i}=\frac{1}{c_{i}} \sum_{j} G_{i j} B_{j}$, so that $A_{i}$ is a self-adjoint operator squaring to the identity. From Lemma 4.1 we can conclude that

$$
c_{i}\left(A_{i} \otimes I\right)|\psi\rangle=\sum_{j} G_{i j}\left(I \otimes B_{j}\right)|\psi\rangle
$$

and hence

$$
\sum_{i, j} G_{i j}\left\langle\psi\left|A_{i} \otimes B_{j}\right| \psi\right\rangle=\sum_{i} c_{i}
$$

is the optimal success bias for $G$. Thus $\left\{A_{i}\right\},\left\{B_{j}\right\},|\psi\rangle$ is an optimal quantum strategy for $G$. It is non-degenerate because $\operatorname{cl} \lambda H=H$ and $\lambda^{*}=\lambda$.

Now we can give the proof of Corollary 2.4.

Proof of Corollary 2.4. Let $\left\{B_{j}\right\}, \rho$ be a cyclic representation of $\mathcal{A}$ on a Hilbert space $H$ such that $\rho$ commutes with all $B_{j}$. Let $\alpha_{i}$ be the eigenvectors of $\rho$. By the spectral theorem for self-adjoint compact operators (see [21] for the statement of this theorem), $H$ decomposes into a direct sum of finite-dimensional eigenspaces $E_{\alpha_{i}}$ of $\rho$. If $v \in E_{\alpha_{i}}$ is an eigenvector, then $\rho B_{j} v=B_{j} \rho v=\alpha_{i} B_{j} v$, and hence $\mathcal{A}$ preserves the eigenspaces of $\rho$. Because each $E_{\alpha_{i}}$ is finite-dimensional, each decomposes further into a sum of irreducible representations of $\mathcal{A}$. Thus we can conclude that $H$ decomposes into a direct sum of finite-dimensional irreducible representations $H_{k}$.

Now we can write $\rho$ as a sum of density operators which have orthogonal support. Namely, let $\rho_{k}=I / \operatorname{tr}(I)$, where $I$ denotes the identity on $H_{k}$. Then $\rho=\sum p_{k} \rho_{k}$, where the $p_{k}$ 's are positive scalars summing to one. By Theorem 11.8 of [14], $S(\rho)=\sum p_{k} S\left(\rho_{k}\right)+H\left(p_{k}\right)$, where $S$ denotes the von Neumann entropy and $H$ denotes the Shannon entropy. $H\left(p_{k}\right)>0$ unless there is exactly one invariant subspace. Hence the minimum entanglement will be attained only by an irreducible representation. The entanglement used by an irreducible representation is $S\left(\rho_{k}\right)=$ $\log _{2}\left(\operatorname{dim} H_{k}\right)$.

\section{Quantum strategies: the approximate case}

The goal of this section is to prove Theorem [2.12. We rely on the following lemma, which is a concatenation of lemmas concerning eigenvalue gaps due to Babai and Friedl.

Lemma 5.1 (Babai and Friedl, 1991). Let $\rho$ be a density matrix on the finite dimensional Hilbert space $\mathbb{C}^{d}$ such that $\tau:=\|\rho-\mathbb{1} / d\|>0$. Then there is an orthogonal decomposition $\mathbb{C}^{d}=W_{1} \oplus W_{2}$ such that

- $W_{1}$ and $W_{2}$ are non-trivial invariant subspaces of $\rho$,

- if $\alpha_{1}$ and $\alpha_{2}$ are eigenvalues of $\rho$ with eigenvectors in $W_{1}$ and $W_{2}$ respectively, then $\alpha_{1} \geq$ $\alpha_{2}+\tau / d$, and 
- if $\rho$ approximately commutes with another matrix $S$, meaning that $\|\rho S-S \rho\| \|_{F} \leq \epsilon$, then

$$
\left\|P_{1} S P_{1}+P_{2} S P_{2}-S\right\|_{F} \leq \frac{\epsilon d}{\tau}
$$

where $P_{i}$ is the orthogonal projection onto $W_{i}$.

Proof. Write the eigenvalues of $\rho$ in descending order as $\lambda_{1} \geq \ldots \geq \lambda_{d}$. Since $\sum \lambda_{i}=1$, we must have $\lambda_{1} \geq 1 / d \geq \lambda_{n}$. Let $\Delta=\max \left|\lambda_{i}-\lambda_{i+1}\right|$ be the largest eigenvalue gap. Then

$$
\tau=\max \left|\lambda_{i}-1 / d\right| \leq \lambda_{1}-\lambda_{d} \leq d \Delta,
$$

so $\Delta \geq \tau / d$. Then apply Lemma 2.11 and Remark 2.7 of [1].

Proof of Theorem 2.12. We want to find a projection $P$ on $H_{2}$ such that $\left\|P-\left(P B_{j} P\right)^{2}\right\|$ and $\left\|c_{i}^{2} P-\left(\sum_{l} G_{i l} P B_{l} P\right)^{2}\right\|$ are bounded above by $C d^{5 / 3} \epsilon^{1 / 12}$ for all $1 \leq i \leq m$ and $1 \leq j \leq n$. We start by bounding $\left\|c_{i}^{2} P-\left(\sum_{l} G_{i l} P B_{l} P\right)^{2}\right\|$. Let $C^{\prime}=\sqrt{10}(m+n)^{1 / 4}$.

Let $|\psi\rangle=\sum|i\rangle \lambda|i\rangle$ and $\rho=\lambda \lambda^{*}$ be the partial trace of $|\psi\rangle$ with respect to $H_{1}$. If $\left\{A_{i}\right\},\left\{B_{j}\right\}$, $|\psi\rangle$ is $\epsilon$-optimal, then by Theorem 3.1, $\|\left(c_{i} A_{i} \otimes \mathbb{1}-\sum_{j} G_{i j} \mathbb{1} \otimes B_{j}\right)|\psi\rangle \| \leq C^{\prime} \epsilon^{1 / 4}$. It follows from Lemma 4.1 that $\left\|c_{i} \lambda \bar{A}-\sum_{j} G_{i j} B_{j} \lambda\right\|_{F} \leq C^{\prime} \epsilon^{1 / 4}$. It follows that $\left\|c_{i}^{2} \rho-\sum_{j} G_{i j} B_{j} \rho \sum_{j} G_{i j} B_{j}\right\| \leq$ $3 C^{\prime} \epsilon^{1 / 4}$. But Lemma 4.1 also tells us that $\left\|\rho \sum_{j} G_{i j} B_{j}-\sum_{j} G_{i j} B_{j} \rho\right\|_{F} \leq 2 C^{\prime} \epsilon^{1 / 4}$ for all $j$, so combining this with the last inequality, we get

$$
\left\|\left(c_{i}^{2}-\left(\sum_{j} G_{i j} B_{j}\right)^{2}\right) \rho\right\| \leq 5 C^{\prime} \epsilon^{1 / 4} .
$$

Inequality (2) is potentially much weaker than the inequality we want, since $\rho$ might have arbitrarily small eigenvalues. Lemma 5.1 suggests two ways to strengthen this inequality. The first is to replace $\rho$ with the maximally mixed density matrix: let $\tau=\|\rho-\mathbb{1} / d\|$ and observe that

$$
\begin{aligned}
\left\|c_{i}^{2}-\left(\sum_{j} G_{i j} B_{j}\right)^{2}\right\| & \leq d\left\|\left(c_{i}^{2}-\left(\sum_{j} G_{i j} B_{j}\right)^{2}\right) \rho\right\| \\
& +d\left\|c_{i}^{2}-\left(\sum_{j} G_{i j} B_{j}\right)^{2}\right\|\|\rho-\mathbb{1} / d\| \leq 5 C^{\prime} d \epsilon^{1 / 4}+2 d \tau .
\end{aligned}
$$

If $\tau=0$ then we can take $P=\mathbb{1}$ and we will be done. If $\tau>0$ then we can apply Lemma 5.1 to decompose $H_{2}$ as $W_{1} \oplus W_{2}$. Let $P_{i}$ denote the orthogonal projection onto $W_{i}$. Let $B_{j}^{\prime}=$ $P_{1} B_{j} P_{1}+P_{2} B_{j} P_{2}$ and let $\rho_{i}=P_{i} \rho P_{i}$. Because $\sum_{j} G_{i j} B_{j}$ approximately commutes with $\rho$, we get $\left\|\sum_{j} G_{i j} B_{j}-\sum_{j} G_{i j} B_{j}^{\prime}\right\| \leq 2 C^{\prime} d \epsilon^{1 / 4} / \tau$. If we let $D_{k l}=\sum_{j} G_{i j} P_{k} B_{j} P_{l}$, then

$$
\sum_{j} G_{i j} B_{j}-\sum_{j} G_{i j} B_{j}^{\prime}=\left(\begin{array}{cc}
0 & D_{12} \\
D_{21} & 0
\end{array}\right),
$$

so $\left\|D_{12}\right\|,\left\|D_{21}\right\| \leq 2 C^{\prime} d \epsilon^{1 / 4} / \tau$, and

$$
\left\|\left(c_{i}^{2} P_{1}-D_{11}^{2}-D_{12} D_{21}\right) \rho_{1}\right\| \leq 5 C^{\prime} \epsilon^{1 / 4} .
$$


But $\left\|D_{12} D_{21}\right\| \leq 4 C^{\prime} d^{2} \epsilon^{1 / 2} / \tau^{2}$, so

$$
\left\|\left(c_{i}^{2}-D_{11}^{2}\right) \rho_{1}\right\| \leq 5 C^{\prime} \epsilon^{1 / 4}+4\left(C^{\prime}\right)^{2} d^{2} \epsilon^{1 / 2} / \tau^{2} .
$$

Since $\rho_{1}$ has minimum eigenvalue at least $\tau / d$, we can conclude that

$$
\begin{aligned}
\left\|c_{i}^{2} P_{1}-\left(\sum_{j} G_{i j} P_{1} B_{j} P_{1}\right)^{2}\right\| & \leq\left\|\left(c_{i}^{2} P_{1}-\left(\sum_{j} G_{i j} P_{1} B_{j} P_{1}\right)^{2}\right) \rho_{1}\right\|\left\|\left(\rho_{1}\right)^{-1}\right\| \\
& \leq \frac{5 C^{\prime} d \epsilon^{1 / 4}}{\tau}+\frac{4\left(C^{\prime}\right)^{2} d^{3} \epsilon^{1 / 2}}{\tau^{3}} .
\end{aligned}
$$

This leaves us to compare the efficacy of two choices for the projection $P$ required by the proposition. If we choose $P=\mathbb{1}$, then we have an upper bound on $\left\|c_{i}^{2} P-\left(\sum_{j} G_{i j} P B_{j} P\right)^{2}\right\|$ given by $f_{1}(\tau)=5 C^{\prime} d \epsilon^{1 / 4}+2 d \tau$, while if we choose $P=P_{1}$ we have an upper bound given by $f_{2}(\tau)=5 C^{\prime} d \epsilon^{1 / 4} / \tau+4\left(C^{\prime}\right)^{2} d^{3} \epsilon^{1 / 2} / \tau^{3}$. Let $\tau_{0}=\frac{3}{2}\left(C^{\prime}\right)^{1 / 2} d^{1 / 2} \epsilon^{1 / 8}$, and observe that $f_{1}\left(\tau_{0}\right)$ and $f_{2}\left(\tau_{0}\right)$ are both bounded above by $8\left(C^{\prime}\right)^{1 / 2} d^{3 / 2} \epsilon^{1 / 8}$. Since $f_{1}$ is increasing, if $\tau \leq \tau_{0}$ then we can take $P=\mathbb{1}$ and get the inequality required by the proposition. If $\tau>\tau_{0}$, then $f_{2}$ is decreasing on the interval $(0, \infty)$, so we can take $P=P_{1}$ and get the inequality required by the proposition.

So far we have ignored the inequality we are supposed to get on $\left\|P-\left(P B_{j} P\right)^{2}\right\|$. If we take $P=\mathbb{1}$, then $P=\left(P B_{j} P\right)^{2}$. So suppose $\tau>\tau_{0}$ and we take $P=P_{1}$. Now by Theorem 3.1 applied to the columns, $\|\left(d_{j} \mathbb{1} \otimes B_{j}-\sum_{i} G_{i j} A_{i} \otimes \mathbb{1}\right)|\psi\rangle \| \leq C^{\prime} \epsilon^{1 / 4}$. Hence $\left\|B_{j} \rho-\rho B_{j}\right\|_{F} \leq 2 C^{\prime} \epsilon^{1 / 4} / d_{j}$. As before, Lemma 5.1 tells us that $\left\|P_{1} B_{j} P_{2}\right\|,\left\|P_{2} B_{j} P_{1}\right\| \leq 2 C^{\prime} d \epsilon^{1 / 4} /\left(d_{j} \tau\right)$, so

$$
\left\|P_{1}-\left(P_{1} B_{j} P_{1}\right)^{2}\right\| \leq \frac{4\left(C^{\prime}\right)^{2} d^{2} \epsilon^{1 / 2}}{d_{j}^{2} \tau^{2}} .
$$

Let $f_{3}(\tau)=4\left(C^{\prime}\right)^{2} d^{2} \epsilon^{1 / 2} /\left(d_{j}^{2} \tau^{2}\right)$. Then $f_{3}$ is decreasing and

$$
f_{3}\left(\tau_{0}\right)=\frac{8 C^{\prime} d \epsilon^{1 / 4}}{9 d_{j}^{2}} \leq\left(C^{\prime}\right)^{1 / 2} d \epsilon^{1 / 8},
$$

where the last inequality comes from the hypothesis that $\epsilon \leq d_{j}^{16} /\left(C^{\prime}\right)^{4}$. So we get $f_{3}(\tau) \leq$ $8\left(C^{\prime}\right)^{1 / 2} d^{3 / 2} \epsilon^{1 / 8}$ as long as $\tau>\tau_{0}$.

\section{Stable relations for the Clifford algebra}

We use the following stability result for amenable groups due to Kazhdan to prove that the relations defining the Clifford algebra are stable.

Definition 6.1. An $\epsilon$-representation of a topological group $G$ is a continuous map $\phi$ from $G$ to the group of unitary transformations on a Hilbert space, such that

$$
\left\|\phi\left(g g^{\prime}\right)-\phi(g) \phi\left(g^{\prime}\right)\right\| \leq \epsilon \text { for all } g, g^{\prime} \in G .
$$

Here $\|\cdot\|$ is the operator norm.

Theorem 6.2 (Kazhdan, Theorem 1 of [12]). Let $G$ be an amenable group and $\phi: G \rightarrow U$ an $\epsilon$-representation of $G$, for some $0<\epsilon<1 / 100$. Then there is a representation $\pi: G \rightarrow U$ such that $\|\phi(g)-\pi(g)\| \leq \epsilon$ for all $g \in G$. 
Let $G_{n}$ be the multiplicative subgroup generated by the elements $Y_{1}, \ldots, Y_{n}$ in the Clifford algebra $\mathcal{C}_{n}$. As a set, $G_{n}$ is equal to

$$
\left\{J^{a_{0}} Y_{0}^{a_{1}} \cdots Y_{n}^{a_{n}}: a_{0}, \ldots, a_{n} \in\{0,1\}\right\}
$$

where we use $J$ to denote $-\mathbb{1}$ for clarity. $G_{n}$ is a finite group of order $2^{n+1}$, and hence is an amenable group with the discrete topology.

Lemma 6.3. Suppose $B_{1}, \ldots, B_{n}$ is an $\epsilon$-approximate representation of $\mathcal{C}_{n}$ on the finite-dimensional Hilbert space $\mathbb{C}^{d}$, where $0<\epsilon<1 /\left(250 n^{2}\right)$. Then there is a representation $B_{1}^{\prime}, \ldots, B_{n}^{\prime}$ of $\mathcal{C}_{n}$ on $\mathbb{C}^{d}$ such that $\left\|B_{i}-B_{i}^{\prime}\right\| \leq 5 n^{2} \epsilon / 2$.

Proof. Since $B_{i}$ is self-adjoint and $\left\|B_{i}^{2}-\mathbb{1}\right\| \leq \epsilon$, every eigenvalue of $B_{i}$ is within $\epsilon$ of either +1 or -1 . Hence there is a self-adjoint unitary matrix $\hat{B}_{i}$ such that $\left\|B_{i}-\hat{B}_{i}\right\| \leq \epsilon$. Since $\left\|B_{i} B_{j}+B_{j} B_{i}\right\| \leq \epsilon$, it follows that $\left\|\hat{B}_{i} \hat{B}_{j}+\hat{B}_{j} \hat{B}_{i}\right\| \leq 5 \epsilon$. Suppose $J^{a_{0}} Y_{1}^{a_{1}} \cdots Y_{n}^{a_{n}} \cdot J^{b_{0}} Y_{1}^{b_{1}} \cdots Y_{n}^{b_{n}}=$ $J^{c_{0}} Y_{1}^{c_{1}} \cdots Y_{n}^{c_{n}}$. This means that the product $J^{a_{0}} Y_{1}^{a_{1}} \cdots Y_{n}^{a_{n}} \cdot J^{b_{0}} Y_{1}^{b_{1}} \cdots Y_{n}^{b_{n}}$ can be transformed to $J^{c_{0}} Y_{1}^{c_{1}} \cdots Y_{n}^{c_{n}}$ using the relations $Y_{i} Y_{j}=-Y_{j} Y_{i}$ at most $n(n-1) / 2$ times. So the map

$$
\psi: J^{a_{0}} Y_{1}^{a_{1}} \cdots Y_{n}^{a_{n}} \mapsto(-1)^{a_{0}} \hat{B}_{1}^{a_{1}} \cdots \hat{B}_{n}^{a_{n}}
$$

gives a unitary $\epsilon^{\prime}$-representation of $G_{n}$, where $\epsilon^{\prime}=5\left(\begin{array}{l}n \\ 2\end{array}\right) \epsilon$. By Kazhdan's theorem, there is a representation $\phi$ of $G_{n}$ with $\|\phi-\psi\| \leq \epsilon^{\prime}$ and $\left\|\phi\left(Y_{i}\right)-B_{i}\right\| \leq \epsilon^{\prime}+\epsilon \leq 5 n^{2} \epsilon / 2$. Let $B_{i}^{\prime}=\phi\left(Y_{i}\right)$. Now $B_{i}^{\prime} B_{j}^{\prime}=\phi(J) B_{j}^{\prime} B_{i}^{\prime}$, so we will be done if we can show that $\phi(J)=-\mathbb{1}$. But $\psi(J)=-\mathbb{1}$, so $\|\psi(J)+\mathbb{1}\| \leq \epsilon^{\prime}<1$. Since $\psi(J)$ is unitary and squares to $\mathbb{1}$, it follows that $\psi(J)=-\mathbb{1}$.

Proof of Proposition 2.11. Let $E^{k l}$ denote the $n \times n$ matrix with ones in the $k l$ th and $l k$ th positions, and zeroes everywhere else. Map every $m \times n$ XOR non-local game $G$ to an $m$-tuple of rank one $n \times n$ matrices $G^{1}, \ldots, G^{m}$, where $G_{k l}^{i}=G_{i k} G_{i l}$. If the $i$ th row of $G$ is equal to the vector with ones in the $l$ th and $k$ th positions and zeroes elsewhere, then $G^{i}$ will be equal to $E^{k k}+E^{l l}+E^{k l}$. Thus whenever $m \geq\left(\begin{array}{l}n \\ 2\end{array}\right)$, there is at least one XOR non-local game such that the matrices $G^{1}, \ldots, G^{m}$, $E^{11}, \ldots, E^{n n}$ span the space of $n \times n$ symmetric matrices. We can think of the space of $m \times n$ XOR non-local games either as $\mathbb{R}^{m n}$ (without normalization) or as the $m n-1$-sphere in $\mathbb{R}^{m n}$ (with normalization). In either case, the subset of XOR non-local games for which the matrices $G^{1}, \ldots, G^{m}, E^{11}, \ldots, E^{n n}$ are linearly dependent is an algebraic set. Hence for almost all $m \times n$ games the matrices $G^{1}, \ldots, G^{m}, E^{11}, \ldots, E^{n n}$ span the space of symmetric matrices. Assume that $G$ is a game for which this is true.

Let $M$ be the $n \times n$ matrix with coefficients $M_{i j}=X_{i} X_{j}$ in $\mathcal{A}$. The defining relations for $\mathcal{A}$ can be rewritten in this notation as

$$
E^{j j} \cdot M=\mathbb{1}_{\mathcal{A}} \text { and } G^{i} \cdot M=c_{i}^{2} \cdot \mathbb{1}_{\mathcal{A}},
$$

where $X \cdot Y$ denotes the coordinate-wise $\mathcal{A}$-valued scalar product. For every $1 \leq k, l \leq n$ there are scalars $s_{j}$ and $t_{i}$ such that

$$
\sum_{j} s_{j} E^{j j}+\sum t_{i} G^{i}=E^{k l}
$$

Let $V_{k l}=\sum_{i} t_{i} c_{i}^{2}+\sum_{j} s_{j}$. Then

$$
\begin{aligned}
X_{k} X_{l}+X_{l} X_{k}-V_{k l} \mathbb{1}_{\mathcal{A}} & =E^{k l} \cdot M-V_{k l} \\
& =\sum_{j} s_{j}\left(E^{j j} \cdot M-\mathbb{1}_{\mathcal{A}}\right)+\sum_{i} t_{i}\left(G^{i} \cdot M-c_{i}^{2} \mathbb{1}_{\mathcal{A}}\right) .
\end{aligned}
$$


So for almost all $m \times n$ games the solution algebra is strongly Clifford.

Now assume that $\mathcal{A}$ is strongly Clifford. There are self-adjoint elements $Y_{1}, \ldots, Y_{r} \in \mathcal{A}$ satisfying the usual Clifford relations, where $r=\operatorname{rank} V$. Each $Y_{i}$ is a linear combination $\sum y_{i j} X_{j}$ of the $X_{j}$ 's, and $2 \delta_{i k}=Y_{i} Y_{k}+Y_{k} Y_{i}=\sum_{j, l} y_{i j} y_{k l}\left(X_{j} X_{l}+X_{l} X_{j}\right)=\sum_{j, l} y_{i j} y_{k l} V_{j l}$. Suppose $\left(B_{1}, \ldots, B_{n}\right)$ is an $\epsilon$-approximate representation of $\mathcal{A}$, and let $\tilde{Y}_{i}=\sum y_{i j} B_{j}$. Then

$$
\tilde{Y}_{i} \tilde{Y}_{k}+\tilde{Y}_{k} \tilde{Y}_{i}-2 \delta i j=\sum_{j, l} y_{i j} y_{k l}\left(B_{j} B_{l}+B_{l} B_{j}-V_{j l} \mathbb{1}\right) .
$$

The relations $X_{j} X_{l}+X_{l} X_{j}-V_{j l} \mathbb{1}$ are in turn given by equation (3) for some choice of constants $s_{j}$ and $t_{i}$. It follows that $\left\|\tilde{Y}_{i} \tilde{Y}_{k}+\tilde{Y}_{k} \tilde{Y}_{i}-2 \delta_{i j}\right\| \leq D^{\prime} \epsilon$ for some (possibly large) constant $D^{\prime}$. In

particular $\left\|\tilde{Y}_{i}\right\| \leq 1+D^{\prime} \epsilon / 4$. If we assume $D^{\prime} \epsilon \leq 1$ then we can truncate eigenvalues of $\tilde{Y}_{i}$ to 1 to get a $5 D^{\prime} \epsilon / 2$-approximate representation of $C_{r}$. Take $\delta=1 /\left(625 D^{\prime} r^{2}\right)$. If $\epsilon<\delta$ then $\epsilon D^{\prime}<1$ and $5 D^{\prime} \epsilon / 2<1 /\left(250 r^{2}\right)$, so we can apply the above lemma to get an exact representation.

\section{Examples}

\subsection{XOR non-local games constructed from a graph}

There is a general method which allows us to construct many examples of games requiring high entanglement. Start with a graph $G$ with $v$ vertices and $e$ edges. We construct a matrix $A$ which has two rows for each edge of $G$, and columns indexed by the vertices. If $i j$ is an edge in $G$ with $i<j$, then the first row corresponding to $i j$ will contain a 1 in the $i$ th column, a -1 in the $j$ th column, and zeroes everywhere else. The other row will contain a 1 in both the $i$ th and the $j$ th column, with zeroes everywhere else. Let $A_{G}$ be the XOR non-local game with game matrix $A / 4 e$.

For example, if $K_{2}$ is the complete graph on 2 vertices, and $G$ is the graph with 3 vertices and edge set $\{12,13\}$, then

$$
A_{K_{2}}=\frac{1}{4}\left(\begin{array}{cc}
1 & -1 \\
1 & 1
\end{array}\right) \text { and } A_{G}=\frac{1}{8}\left(\begin{array}{ccc}
1 & -1 & 0 \\
1 & 1 & 0 \\
1 & 0 & -1 \\
1 & 0 & 1
\end{array}\right)
$$

Proposition 7.1. Let $A_{G}$ be the $2 e \times v$ game constructed from the graph $G$ according to the above prescription. Then the optimal solutions to the associated convex programming problem $(\Gamma)$ of Proposition 3.3 are the semidefinite matrices $V$ satisfying the conditions

$$
V_{i i}=1 \text { for all } 1 \leq i \leq v \text { and } V_{i j}=0 \text { for each edge ij of } G \text {. }
$$

The optimal quantum bias for $A_{G}$ will be $1 / 2$ and the optimal classical bias will be $1 / \sqrt{2}$. The solution algebra for $A_{G}$ will be the $C^{*}$-algebra generated by $X_{1}, \ldots, X_{v}$ satisfying the relations

$$
X_{i}^{2}=\mathbb{1} \text { for all } 1 \leq i \leq v \text { and } X_{i} X_{j}=-X_{j} X_{i} \text { for each edge ij of } G .
$$

Proof of Proposition 7.1. For convenience, let $f(t)$ denote the function $\sqrt{1+t}+\sqrt{1-t}$. The optimization problem $(\Gamma)$ has objective function

$$
\sum_{i j \in E(G)} \frac{1}{2 \sqrt{2} e} f\left(V_{i j}\right),
$$


using the fact that $V_{i i}=1$ at any feasible point. Now $f(0)=2$, and this is the unique maximum of $f(t)$ on the interval $[-1,1]$. If $V$ is feasible for $(\Gamma)$ then $V_{i j} \in[-1,1]$, so $(\Gamma)$ has optimum value $1 / \sqrt{2}$, and the optimal solutions are the feasible solutions $V$ with $V_{i j}=0$ if $i j \in E(G)$.

On the other hand, if $V$ corresponds to a classical solution, then $V_{i j}= \pm 1$, and $f( \pm 1)=\sqrt{2}$.

If $V$ is optimal for $(\Gamma)$ then the squares of the two row biases corresponding to edge $i j$ are

$$
c=\frac{\sqrt{\left(V_{i j}^{2} \pm 2 V_{i j}^{2}+V_{j j}^{2}\right)}}{4 e}=\frac{1}{2 \sqrt{2} e} .
$$

Given that $X_{i}^{2}=X_{j}^{2}=\mathbb{1}$, the relations

$$
\left(\frac{X_{i}}{4 e} \pm \frac{X_{j}}{4 e}\right)^{2}=c^{2} \mathbb{1}
$$

are equivalent to the single relation $X_{i} X_{j}=-X_{j} X_{i}$.

The game described by $A_{K_{2}}$ is commonly known as the CHSH game. As an obvious generalization of the $\mathrm{CHSH}$ game, we define $\mathrm{CHSH}(n)$ to be the XOR game with game matrix $A_{K_{n}}$. The associated solution algebra is strongly Clifford of rank $n$, and every irreducible representation of the solution algebra has dimension $2^{\lfloor n / 2\rfloor}$. Thus Corollaries 2.4 and 2.13 give lower bounds on the entanglement required for optimal and near-optimal strategies. For $\mathrm{CHSH}(n)$ we also can derive a lower bound on the rank of near-optimal vector strategies.

Proposition 7.2. Let $\epsilon>0$. Every $\epsilon$-optimal vector strategy for $\operatorname{CHSH}(n)$ is supported on a Hilbert space of dimension at least $n-8 \sqrt{2} n(n-1) \epsilon$.

Proof. For the first part of the Proposition, let $\left\{u_{i j l}\right\}_{l \in\{0,1\}},\left\{v_{j}\right\}$ be an $\epsilon$-optimal vector strategy. Let $V$ be the matrix with $V_{i j}=v_{i} \cdot v_{j}$. Observe that

$$
\sum_{1 \leq i<j \leq n} \frac{1}{\sqrt{2} n(n-1)} f\left(V_{i j}\right) \geq \sum_{1 \leq i<j \leq n} \frac{1}{2 n(n-1)} u_{i j 0}\left(v_{i}-v_{j}\right)+\frac{1}{2 n(n-1)} u_{i j 1}\left(v_{i}+v_{j}\right) \geq \frac{1}{\sqrt{2}}-\epsilon
$$

where $f(t)=\sqrt{1+t}+\sqrt{1-t}$ again. Using the Taylor series expansion, we see that $f(t) \leq 2-t^{2} / 4$ for all $t \in[-1,1]$. Consequently

$$
\sum_{1 \leq i<j \leq n} \frac{1}{\sqrt{2} n(n-1)} f\left(V_{i j}\right) \leq \frac{1}{\sqrt{2}}-\frac{\|V-I\|_{F}^{2}}{8 \sqrt{2} n(n-1)} .
$$

Now $\operatorname{dim} \operatorname{span}_{\mathbb{R}}\left\{v_{j}\right\}=\operatorname{rank} V=n-z$, where $z$ is the number of eigenvalues of $V-I$ equal to -1 . But $\|V-I\|_{F}^{2} \geq z$, implying that

$$
n-z \geq n-\|V-I\|_{F}^{2} \geq n-8 \sqrt{2} n(n-1) \epsilon .
$$

\subsection{A game for which Tsirelson's bound is tight}

$\operatorname{Because} \operatorname{CHSH}(n)$ is an $n(n-1) \times n$ game, $\operatorname{CHSH}(n)$ does not quite meet Tsirelson's upper bound on entanglement. To give a game which does meet Tsirelson's upper bound (for $n \geq 2$ ), let 
$m=n(n-1) / 2$. Define a matrix $G$, with rows indexed by pairs $i j$, where $1 \leq i<j \leq n$, and columns indexed by the set $\{1, \ldots, n\}$, as

$$
G_{i j, k}= \begin{cases}1 / 2 m & i=k \\ -1 / 2 m & j=k \\ 0 & \text { otherwise }\end{cases}
$$

Then $G$ defines an $m \times n$ XOR non-local game. Now look at the optimization problem $(\Gamma)$ for this game. The objective function of $(\Gamma)$ is invariant under conjugation of $V$ by permutation matrices. Thus we can solve $(\Gamma)$ by the standard technique of summing over the action of the symmetric group to reduce to a linear program. It follows that $V=\left(2 m^{2} s^{2}\right) I+\left(1-2 m^{2} s^{2}\right) E$ is a solution to $(\Gamma)$, where $E$ is the matrix of all ones. The marginal row biases can be determined by the formula $c_{i j}^{2}=\left(1-V_{i j}\right) / 2 m^{2}$. The solution algebra will be a Clifford algebra of rank $n-1$, and thus $G$ requires $\lfloor(n-1) / 2\rfloor$ ebits.

\subsection{Clifford strategies do not always use minimal entanglement}

To give an example of a game for which the strategy of minimum entanglement is not Clifford, we use the graph construction of Proposition [7.1. Given an integer $n$, let $Y_{1}, \ldots, Y_{n}$ be the canonical generators of the Clifford algebra $\mathcal{C}_{n}$. Define a graph $G$ whose vertices are ordered $k$-tuples $\left(i_{1}, \ldots, i_{k}\right)$, where $1 \leq i_{1}<\ldots<i_{k} \leq n$, and $k$ ranges between 1 and $n$. Place an edge in $G$ between $\left(i_{1}, \ldots, i_{k}\right)$ and $\left(j_{1}, \ldots, j_{l}\right)$ if $Y_{i_{1}} \cdots Y_{i_{k}}$ anticommutes with $Y_{j_{1}} \cdots Y_{j_{l}}$ in $\mathcal{C}_{n}$. Let $C L(n)$ be the XOR non-local game $A_{G}$ constructed from the graph $G$ according to the recipe in Proposition 7.1. We want to show that the optimal strategies of minimum entanglement for $C L(n)$ are not Clifford.

Proposition 7.3. Let $(\Gamma)$ be the convex programming problem associated to $C L(n)$ by Proposition 3.3. For $n \geq 3$, every solution $V$ to $(\Gamma)$ has rank at least $(4 n-8) / 3$.

Proof. We use a pigeon-hole argument. Let $V$ be a solution to $(\Gamma)$, and take unit vectors $v(\tilde{i})$ in $\mathbb{R}^{N}$ such that $V_{\tilde{i}, \tilde{j}}=v(\tilde{i}) \cdot v(\tilde{j})$. For convenience of notation we ignore order in the multi-indices, so for example $v(2,1)$ would be the same as $v(1,2)$. Now $i j$ is an edge in $G$ for every $i \neq j$, and hence $v(i) \cdot v(j)=0$. Let $W=\operatorname{span}\{v(i): 1 \leq i \leq n\}$, and write

$$
\operatorname{span}\{v(\tilde{i})\}=W \oplus W^{\perp} .
$$

It will also be convenient to let

$$
W\left(i_{1}, \ldots, i_{k}\right)=\operatorname{span}\left\{v\left(i_{1}\right), \ldots, v\left(i_{k}\right)\right\} \oplus W^{\perp} .
$$

Now there are $\left(\begin{array}{l}n \\ 3\end{array}\right)$ vectors of the form $v(i j k)$. If $l \notin\{i, j, k\}$ then $v(l) \cdot v(i j k)=0$. Hence $v(i j k)$ is contained in $W(i j k)$, and has non-zero projection onto one of $W(i j), W(i k)$, or $W(j k)$. For $i<j$ let $Z_{i j}$ be the set of vectors $v(i j k)$ which have a non-zero projection onto $W(i j)$ (note that $k$ need not be greater than $i$ and $j$ ). There are $n-2$ vectors which could potentially be in $Z_{i j}$. However if $k \neq l$ then

$$
\left(P_{W(i j)} v(i j k)\right) \cdot\left(P_{W(i j)} v(i j l)\right)=v(i j k) \cdot v(i j l)=0,
$$

where $P_{W(i j)}$ is the orthogonal projection onto $W(i j)$. Thus $Z_{i j}$ contains no more than $\operatorname{dim} W(i j)=$ $2+\operatorname{dim} W^{\perp}$ vectors. Thus we conclude that

$$
\left(\begin{array}{l}
n \\
3
\end{array}\right) \leq\left(2+\operatorname{dim} W^{\perp}\right)\left(\begin{array}{l}
n \\
2
\end{array}\right)
$$


Solving this inequality we find that $\operatorname{dim} W^{\perp} \geq \frac{n-8}{3}$, and hence

$$
\operatorname{dim} \operatorname{span}\{v(\tilde{i})\} \geq n+\frac{n-8}{3}=\frac{4 n-8}{3}
$$

Let $\mathcal{A}$ be the solution algebra associated to $C L(6 t+2)$. Every optimal vector solution to $C L(6 t+2)$ has rank at least $8 t$, so every Clifford representation uses at least $4 t$ ebits. However, it is clear that any irreducible representation of $\mathcal{C}_{6 t+2}$ is also a representation of $\mathcal{A}$, since we can send $X_{i_{1}, \ldots, i_{k}}$ to $i^{s} Y_{i_{1}} \cdots Y_{i_{k}}$, where $s=\left(\begin{array}{c}k \\ 2\end{array}\right)$. Thus $C L(6 t+2)$ requires at most $3 t+1$ ebits. It follows that any Clifford representation uses at least $t-1$ ebits more than what is required.

\section{Acknowledgments}

I thank Richard Cleve, Alex Fink, and Sarvagya Upadhyay for helpful discussions. I also thank Richard Cleve, Andrew Marks, and Sarvagya Upadhyay for comments on the manuscript. This work was supported in part by NSERC.

\section{References}

[1] Babai, L. and Friedl, K. Approximate representation theory of finite groups. Proceedings of 32nd Annual IEEE Symposium on Foundations of Computer Science (FOCS 1991).

[2] J. Bell. On the Einstein-Podolsky-Rosen Paradox. Physics 1 (3):195-200, 1964.

[3] S. Boyd and L. Vandenberghe. Semidefinite programming. SIAM Review, 38(1):49-95, 1996.

[4] G. Brassard. Quantum communication complexity. Foundations of Physics, 33 (11):1593-1616, 2003.

[5] J. Briet, H. Buhrman, B. Toner. A generalized Grothendieck inequality and entanglement in XOR games. arXiv: quant-ph/0901.2009.

[6] N. Brunner, S. Pironio, A. Acin, N. Gisin, A. A. Methot, and V. Scarani. Testing the Hilbert space dimension. Phys. Rev. Lett. 100, 210503, 2008.

[7] J.F. Clauser, M.A. Horne, A. Shimony, and R.A. Holt. Proposed experiment to test local hidden variable theories. Physical Review Letters, 23 (15):880-884, 1969.

[8] R. Cleve, P. Hoyer, B. Toner, and J. Watrous. Consequences and limits of nonlocal strategies. Proceedings of the 19th IEEE Conference on Computational Complexity (CCC 2004), 236-249, 2004.

[9] R. Cleve, W. Slofstra, F. Unger, S. Upadhyay. Perfect Parallel Repetition Theorem for Quantum XOR Proof Systems. Computational Complexity 17 (2): 282-299, 2008.

[10] R. Cleve. Private communication, 2008.

[11] M. Junge, C. Palazuelos, D. Perez-Garca, I. Villanueva, and M.M. Wolf. Unbounded violations of bipartite Bell Inequalities via Operator Space theory. arXiv: quant-ph/0910.4228 
[12] D. Kazhdan. On $\epsilon$-representations. Israel Journal of Mathematics, Vol. 43, No. 4, 315-323, 1982.

[13] T.A. Loring. $C^{*}$-algebras generated by stable relations. J. Funct. Anal. 112, 1993.

[14] M. Nielsen and I. Chuang. Quantum Computation and Quantum Information. Cambridge University Press, 2000.

[15] D. Perez-Garcia, M.M Wolf, C. Palazuelos, I. Villanueva, and M. Junge. Unbounded violation of tripartite Bell inequalities. Communications in Mathematical Physics, v. 279, no. 2, 2008.

[16] S.J. Summers and R.F. Werner. Bell's inequalities and quantum field theory, I. General setting. Journal of Mathematical Physics, 28, 2440-2447, 1987.

[17] B.S. Tsirelson. Quantum analogues of the Bell inequalities. The case of two spatially separated domains. Journal of Soviet Mathematics 36 (4):557-570, 1987.

[18] B.S. Tsirelson. Some results and problems on quantum Bell-type inequalities. Hadronic Journal Supplement 8(4):329-345, 1993.

[19] T. Vertesi and K.F. Pal. Bounding the dimension of bipartite quantum systems. Physical Review A 79, 042106, 2009.

[20] R.F. Werner and M.M. Wolf. Bell Inequalities And Entanglement. Quantum Information and Computation 1 (3):1-25, 2001.

[21] Robert J. Zimmer. Essential Results of Functional Analysis. University of Chicago Press, 1990. 